\title{
FRB 150418: clues to its nature from European VLBI Network and e-MERLIN observations
}

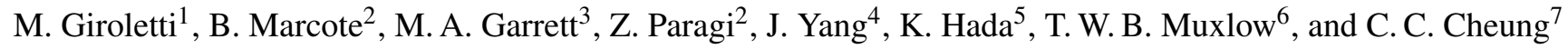 \\ 1 INAF Istituto di Radioastronomia, via Gobetti 101, 40129 Bologna, Italy \\ e-mail: giroletti@ira.inaf.it \\ 2 Joint Institute for VLBI ERIC, Postbus 2, 7990 AA Dwingeloo, The Netherlands \\ 3 Netherlands Institute for Radio Astronomy (ASTRON), Postbus 2, 7990 AA Dwingeloo, The Netherlands \\ 4 Department of Earth and Space Sciences, Chalmers University of Techn., Onsala Space Observatory, 43992 Onsala, Sweden \\ 5 Mizusawa VLBI Observatory, National Astronomical Observatory of Japan, Osawa, Mitaka, Tokyo 181-8588, Japan \\ 6 Jodrell Bank Centre for Astrophysics/e-MERLIN, The University of Manchester, M13 9PL, UK \\ 7 Space Science Division, Naval Research Laboratory, Washington, DC 20375-5352, USA
}

Received 23 June 2016 / Accepted 5 September 2016

\begin{abstract}
Aims. We investigate the nature of the compact and possibly variable nuclear radio source in the centre of WISE J0716-19, the proposed host galaxy of the fast radio burst FRB 150418.

Methods. We observed WISE J0716-19 at 5.0 GHz with the European VLBI Network (EVN) four times between 2016 March 16 and June 2. At three epochs, we simultaneously observed the source with e-MERLIN at the same frequency.

Results. We detected a compact source in the EVN data in each epoch with a significance of up to $\sim 8 \sigma$. The four epochs yielded consistent results within their uncertainties for the peak surface intensity and positions. The mean values for these quantities are $I_{\text {peak }}=(115 \pm 9) \mu \mathrm{Jy}_{\text {beam }}^{-1}$ and RA $=07^{\mathrm{h}} 16^{\mathrm{m}} 34.55496(7)^{\mathrm{s}}$, Dec $=-19^{\circ} 00^{\prime} 39.4754(8)^{\prime \prime}$. The e-MERLIN data provided $\sim 3-5 \sigma$ detections at a position consistent with those of the EVN data. The emission on angular scales intermediate between the EVN and e-MERLIN is consistent with being null. The brightness temperature of the EVN core is $T_{\mathrm{b}} \gtrsim 10^{8.5} \mathrm{~K}$, close to the value previously required to explain the short-term radio variability properties of WISE J0716-19 in terms of interstellar scintillation.

Conclusions. Our observations provide direct and independent evidence of a nuclear compact source in WISE J0716-19, a physical scenario without evident connection with FRB 150418. However, the EVN data do not indicate the variability observed with the VLA.
\end{abstract}

Key words. galaxies: active - galaxies: individual: WISE J071634.59-190039.2 - radio continuum: galaxies - scattering

\section{Introduction}

Fast radio bursts (FRBs) are transient episodes characterised by their short (sub-ms) duration and large dispersion measure (DM). After the initial discovery by Lorimer et al. (2007), several new such events have been discovered (e.g. Thornton et al. 2013; Champion et al. 2016), triggering a debate about their nature. It is possible that these bursts are generated by young, highly magnetized neutron stars, as suggested for the repeating FRB 121102 (Spitler et al. 2016), or by cataclysmic events.

Both Galactic and extragalactic origins have been proposed. An extragalactic origin is preferred based on the large DM, $\gtrsim(0.5-1) \times 10^{3} \mathrm{~cm}^{-3}$ pc as typically found, but only a precise localisation and a measurement of the redshift can help to conclude about this. For this reason, the reported localisation of FRB 150418 to the elliptical galaxy, WISE J071634.59-190039.2 (hereafter, WISE J0716-19) by Keane et al. (2016, hereafter, K16) has attracted interest in the community. The precise redshift determination for this FRB has immediate implications for the system's energetics, thus possible FRB progenitors (Liu et al. 2016; Zhang 2016), as well as applications to probe fundamental physics (Bonetti et al. 2016; Tingay \& Kaplan 2016).
The proposed identification of FRB 150418 with WISEJ0716-19 was based on the prompt detection (beginning $2 \mathrm{hr}$ after the FRB discovery) with the Australia Telescope Compact Array (ATCA) of a fading radio source within one beam of the $21 \mathrm{~cm}$ Parkes multi-beam receiver. Optical photometric and spectroscopic follow-up observations with the Subaru telescope identified an elliptical galaxy at $z=0.492 \pm 0.008$ consistent with the radio source within the $\sim 1^{\prime \prime}$ positional uncertainty. In more detail, the radio transient emission was observed in only the first two epochs of ATCA follow-up separated by six days (flux densities $\sim 0.2 \mathrm{mJy}$ at $5.5 \mathrm{GHz}$ ) with three subsequent detections of essentially steady emission $(\sim 0.1 \mathrm{mJy}$ at $5.5 \mathrm{GHz})$ that was attributed to the emission from the host galaxy.

This association of the prompt ms-duration emission from the FRB with the variable ATCA source has been questioned. Williams \& Berger (2016a, hereafter WB16a) argued instead that WISE J0716-19 is consistent with being a random active galactic nucleus (AGN) found within the Parkes beam based on the known rate of variable (rather than transient) radio sources, that the steady radio emission component implies a high luminosity more typical of an AGN, and that the radio light curve is inconsistent with the evolution of a standard afterglow. 
Table 1. Log of observations, image parameters, and model fit results.

\begin{tabular}{|c|c|c|c|c|c|c|c|c|c|c|}
\hline \multicolumn{2}{|c|}{ Epoch } & \multicolumn{3}{|c|}{ EVN data } & \multicolumn{6}{|c|}{ e-MERLIN data } \\
\hline $\begin{array}{l}\text { Date in } \\
2016 \\
(1)\end{array}$ & $\begin{array}{l}\text { MJD } \\
(2)\end{array}$ & $\begin{array}{c}\text { HPBW } \\
\left(\operatorname{mas} \times \operatorname{mas}^{\circ}{ }^{\circ}\right) \\
(3)\end{array}$ & $\begin{array}{c}I_{\text {peak }} \\
(\mu \mathrm{Jy} \\
(4)\end{array}$ & $\begin{array}{c}I_{\text {noise }} \\
\left.\mathrm{am}^{-1}\right) \\
(5)\end{array}$ & $\begin{array}{c}S_{\text {5.0,JMFIT }} \\
(\mu \mathrm{Jy}) \\
(6)\end{array}$ & $\begin{array}{c}\text { HPBW } \\
\left(\operatorname{mas} \times \operatorname{mas}^{\circ}{ }^{\circ}\right) \\
(7)\end{array}$ & $\begin{array}{r}I_{\text {peak }} \\
(\mu \mathrm{Jy} \\
(8)\end{array}$ & $\begin{array}{c}I_{\text {noise }} \\
\left.\mathrm{am}^{-1}\right) \\
(9)\end{array}$ & $\begin{array}{c}S_{\text {5.0,JMFIT }} \\
(\mu \mathrm{Jy}) \\
(10)\end{array}$ & $\begin{array}{c}\Delta S_{5.0} \\
(\mu \mathrm{Jy}) \\
(11)\end{array}$ \\
\hline March 16 & 57463. & $10.1 \times 6.2,3.9$ & 12 & 18 & $125 \pm 22$ & & & & & \\
\hline May 10 & 57518.6 & $9.7 \times 6.1,8.7$ & 11 & 14 & & $261 \times 2$ & 16 & 55 & 176 & $40 \pm 60$ \\
\hline May 31 & 57539.6 & $10.9 \times 6.1,-7.5$ & 10 & 16 & & $231 \times 27,11$ & 14 & 48 & $158 \pm 51$ & $40 \pm 55$ \\
\hline June 2 & 57541.6 & $9.3 \times 5.3,1.3$ & 133 & 20 & $125 \pm 32$ & $212 \times 28,10$ & 246 & 52 & $264 \pm 59$ & $140 \pm 70$ \\
\hline
\end{tabular}

Notes. Columns 1, 2: observation date; Cols. 3-6: EVN half-peak beam width (HPBW), naturally weighted image peak brightness and $1 \sigma$ noise level, and results of a 2d Gaussian fit to the image brightness distribution; Cols. 7-10: same as Cols. 3-6, for e-MERLIN data; Col. 11: flux density difference between e-MERLIN and EVN data.

Additional criticism of the proposed association came from the results of a Karl G. Jansky Very Large Array (VLA) observing campaign almost a year after the FRB: in ten total observations at 5.5 and $7.5 \mathrm{GHz}$ spanning 35 days, Williams \& Berger (2016a,b) found variable radio emission at a higher level than the previously observed steady $\sim 0.1 \mathrm{mJy}$ source. Vedantham et al. (2016, hereafter V16) also reported a single-epoch multifrequency VLA observation over the $1-18 \mathrm{GHz}$ frequency range that showed a flat-spectrum radio source consistent with an AGN. Finally, numerical simulations by Akiyama \& Johnson (2016) indicated that the reported light curve is consistent with scintillating radio emission from an AGN core with $T_{\mathrm{b}} \gtrsim 10^{9} \mathrm{~K}$.

A final confirmation of the AGN scenario, plus a relevant contribution from refractive interstellar scintillation, can be obtained from high angular resolution Very Long Baseline Interferometry (VLBI) observations. In this Letter, we therefore report on the results of European VLBI Network (EVN) and e-MERLIN observations of WISE J0716-19. In the following, we describe the observations in Sect. 2, present the results in Sect. 3, and discuss them in Sect. 4.

\section{Observations and data reduction}

We observed WISE J0716-19 four times between 2016 March 16 and June 2 (Table 1) with a subset of the EVN. The participating stations were Effelsberg, Hartebeesthoek, Jodrell Bank (Mark2), Medicina, Noto, Onsala, Torun, Yebes, and a single Westerbork telescope. We observed at 5.0 GHz, with eight $16 \mathrm{MHz}$ wide baseband channels, in dual polarization, and with two-bit sampling. The data were electronically transferred over fibre links to the SFXC correlator at JIVE, where they were correlated in real time with the so-called e-VLBI technique.

We carried out all observations in phase-reference mode, with $2.5 \mathrm{~min}$ scans on the target source bracketed by $1.5 \mathrm{~min}$ scans on the nearby $\left(0.9^{\circ}\right.$ offset $)$ calibrator J0718-1813. Each observation lasted for $\sim 5.5 \mathrm{~h}$, with an on-source time of $\sim 2.4 \mathrm{~h}$. We calibrated visibility amplitudes based on the a priori gain curves and measured system temperatures at each station. Parallactic angle corrections were applied, and we determined instrumental single-band delays using a scan on a strong calibrator. We then determined phase, rates, and residual delays for the phase calibrator. Since the calibrator has a double-component structure, we imaged it with a hybrid mapping procedure and then repeated the fringe-fitting process using the obtained image as the input model. The resulting solutions were applied back to the phase reference source, the target, and the additional check source J0712-1847. Bandpass solutions were then determined by combining all the data for the calibrator. Finally, we carried out one cycle of phase-only and one of phase-and-amplitude self-calibration for the phase reference source and transferred the solutions to the target. A parallel analysis of the check source based on either direct fringe-fitting of its visibility data or phase self-calibration indicated that coherence losses affected the detected ranging by between $20 \%$ and $40 \%$ of the real flux density; this is expected because of the low elevation of the target.

Initially, we imaged the first-epoch data over a large field of $4^{\prime \prime} \times 4^{\prime \prime}$, centred on the WISE coordinates, RA = $07^{\mathrm{h}} 16^{\mathrm{m}} 34.59^{\mathrm{s}}, \mathrm{Dec}=-19^{\circ} 00^{\prime} 39.2^{\prime \prime}$. The overall rms noise was about $25 \mu \mathrm{Jy}$ beam $^{-1}$ (Marcote et al. 2016). Following the report of the VLBA and e-MERLIN localisation by Bassa et al. (2016a, hereafter B16), we imaged a smaller field around their preliminary VLBA-measured position. The local noise for an image produced with the AIPS task IMAGR using ROBUST $=5$ is $18 \mu \mathrm{Jy}_{\text {beam }}{ }^{-1}$. In the following epochs, we reached similar or better noise values, except for the last epoch, in which the most sensitive telescope (Effelsberg) did not provide data for about half of the observation run.

In strict simultaneity with the latter three EVN epochs (same start and end times), we observed the source with e-MERLIN, using six, five, and five stations in each experiment. We observed at $5.0 \mathrm{GHz}$ with four $128-\mathrm{MHz}$-wide channels in dual polarisation. The same phase reference source was used as in the EVN run. The maximum elevation of the source was $18^{\circ}$, which resulted in an elongated restoring beam (axial ratio $\sim 10$, in $\mathrm{PA}=\sim 10^{\circ}$ ). Detailed information is reported in Table 1 .

\section{Results}

In Fig. 1 we show our EVN $5.0 \mathrm{GHz}$ images around the position of the VLBA and e-MERLIN detections reported by B16. The main image shows a $0.3^{\prime \prime} \times 0.25^{\prime \prime}$ field of view based on averaging the images from all epochs. The insets show 60 mas $\times 60$ mas image stamps of the central region from the four individual epochs.

In each of the individual epochs, the source is detected with significances above $6 \sigma$. In Cols. 4-6 of Table 1 we report the image peak brightness, the noise, and the component flux density measured with AIPS task JMFIT. The associated uncertainties were calculated as the quadratic sum of a $1 \sigma$ rms statistical contribution and a $10 \%$ absolute calibration uncertainty; this provides the uncertainty on the relative calibration from epoch to epoch, while the overall scaling due to coherence losses remains unaccounted for. Within these uncertainties, the source flux density is consistent with being constant among epochs; the best-fit coordinates are also consistent to $1 / 10$ of the restoring beam, or less. 


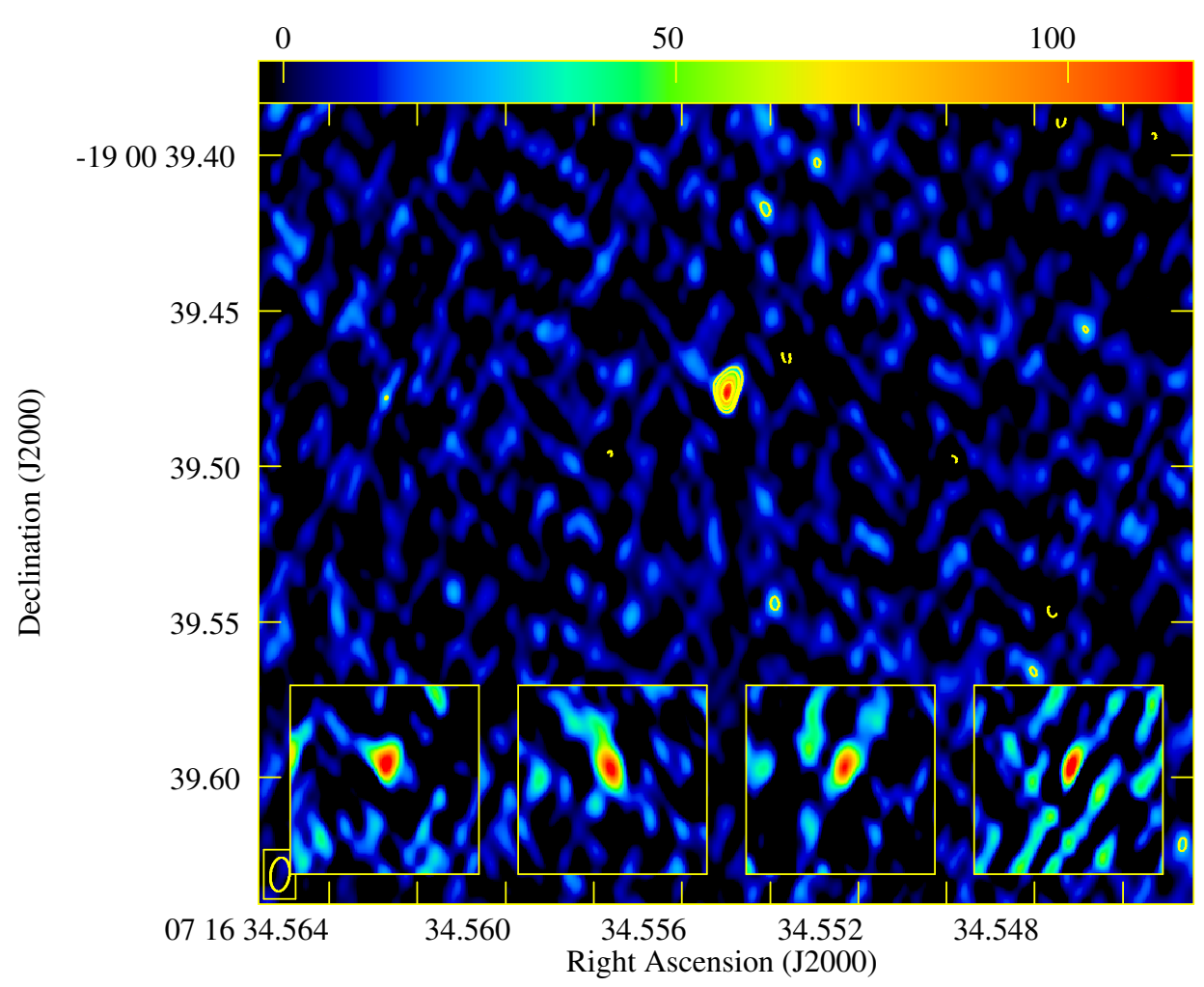

Fig. 1. EVN 5.0 GHz images of WISE J0716-19. Main panel: average image obtained combining all observations; contours are traced at $\pm 3,5,10 \times$ the image local rms noise of $8.9 \mu \mathrm{Jy}_{\text {beam }}{ }^{-1}$; the peak surface brightness is $115 \mu \mathrm{Jy}_{\text {beam }}{ }^{-1}$; the restoring beam is shown in the bottom left corner and it is 10.9 mas $\times 6.1$ mas in PA $=-7^{\circ}$; the colour scale indicates a surface brightness of between -3.0 and $115 \mu \mathrm{Jy} \mathrm{beam}^{-1}$. Insets: central part of the field as obtained from each individual dataset; pixel size and colour scale are the same as in the main panel to facilitate comparison.

Table 2. Normalised EVN peak brightness of target and calibrator in time bins.

\begin{tabular}{lcccccc}
\hline \hline & \multicolumn{3}{c}{ WISE J0716-19 } & \multicolumn{3}{c}{ J1718-1813 } \\
Epoch & $S_{1} /\langle S\rangle$ & $S_{2} /\langle S\rangle$ & $S_{3} /\langle S\rangle$ & $S_{1} /\langle S\rangle$ & $S_{2} /\langle S\rangle$ & $S_{3} /\langle S\rangle$ \\
\hline 1 & $1.13 \pm 0.25$ & $1.14 \pm 0.25$ & $0.74 \pm 0.23$ & $0.93 \pm 0.09$ & $0.99 \pm 0.10$ & $1.08 \pm 0.11$ \\
2 & $0.90 \pm 0.22$ & $0.93 \pm 0.22$ & $1.16 \pm 0.23$ & $0.97 \pm 0.10$ & $1.00 \pm 0.10$ & $1.04 \pm 0.10$ \\
3 & $1.32 \pm 0.30$ & $0.95 \pm 0.29$ & $0.73 \pm 0.28$ & $0.92 \pm 0.09$ & $1.00 \pm 0.10$ & $1.08 \pm 0.11$ \\
4 & $0.88 \pm 0.26$ & $0.53 \pm 0.25$ & $1.60 \pm 0.29$ & $0.92 \pm 0.09$ & $1.02 \pm 0.10$ & $1.05 \pm 0.11$ \\
\hline
\end{tabular}

In the stacked EVN $5.0 \mathrm{GHz}$ image, the peak brightness and the rms noise are $I_{\text {peak }}=115 \mu \mathrm{Jy}_{\text {beam }}{ }^{-1}$ and $I_{\text {noise }}=$ $8.9 \mu \mathrm{Jy}_{\text {beam }}{ }^{-1}$, respectively, amounting to an overall $>12 \sigma$ significance detection. Given the lack of significant variability among epochs, we determined the source parameters from a fit to the mean image, giving $S_{5.0, \mathrm{JMFIT}}=(120 \pm 15) \mu \mathrm{Jy}$, RA $=07^{\mathrm{h}} 16^{\mathrm{m}} 34.55496(7)^{\mathrm{s}}$, Dec $=-19^{\circ} 00^{\prime} 39.4754(8)^{\prime \prime}$ (the digits in parenthesis indicate the standard deviation of the measurements of the four datasets; this position also agrees with that published, during the revision process of this Letter, by Bassa et al. 2016b). At the luminosity distance of WISE J0716-19 $\left(d_{L}=2.81 \mathrm{Gpc}\right)$, the corresponding average monochromatic luminosity is $L_{5.0}=(1.13 \pm 0.15) \times 10^{23} \mathrm{~W} \mathrm{~Hz}^{-1}$.

Our array has a large gap in the $(u, v)$-plane between European baselines and baselines to South Africa; moreover, the phase reference source has a double structure and the phase solutions to Hartebeesthoek are less stable. For these reasons, we determined the above parameters on images based only on the European baseline data. However, the source is still also significantly detected in images with the full array; it is consistent with being unresolved, with a peak brightness $I_{\text {peak }}=$ $118 \mu \mathrm{Jy}$ beam $^{-1}$ and a deconvolved size $\theta<0.34$ mas. We also carried out model-fits using Difmap, fitting a circular Gaussian component to the visibility data. The fit provides a major axis size of 0.18 mas. Taking the visibility plane model-fit size as an upper limit, we estimate a brightness temperature of $T_{\mathrm{b}} \geq$ $10^{8.5} \mathrm{~K}$.

No significant variability is present from one epoch to the other. We explored the presence of variability on shorter timescales by dividing each observation into three bins of about two hours each. The values of the target and the calibrator peak brightness in each bin are reported in Table 2, normalised to the mean of each epoch. The source is generally detected in every subset of the dataset, albeit at lower significance (the relative errors are comparatively large because the noise in each bin is $\sim \sqrt{3} \times$ higher than in the full dataset). In 8 of the 12 cases, the peak brightness is consistent with the mean value. In the last epoch, two bins are about $2 \sigma$ away from the mean. The significance of these variations is difficult to establish because the $(u, v)$-plane coverage is different in every bin, as was the elevation of the source. The calibrator peak brightness is more stable, but generally increases in the second and third subsets of each observation, as the restoring beam rotates closer to the main PA of the double structure of the source. While this indicates that 


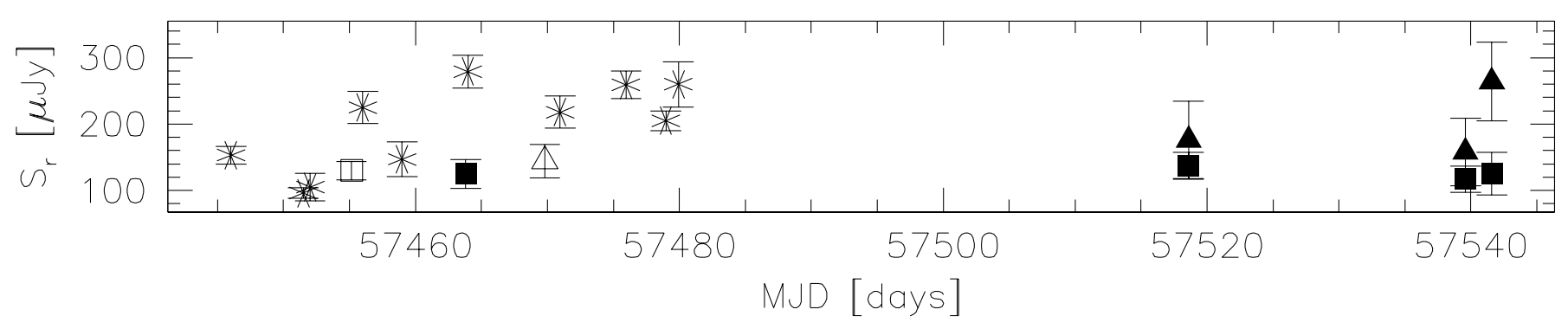

Fig. 2. Light curve of WISE J0716-19. Stars: $5.5 \mathrm{GHz}$ VLA data (V16; WB16a; WB16b); squares: $5.0 \mathrm{GHz}$ VLBI data; triangles: 5.0 GHz e-MERLIN data. The filled symbols are from this work, empty ones from B16.

no systematic effects due to calibration are present, it also shows how difficult it is to establish variability on short timescales with the present data.

Finally, we detect a point-like source in every e-MERLIN observation, at a significance level of just above $3 \sigma$. The coordinates are consistent with those obtained with the EVN, but less well determined because of the elongated beam. The peak brightness in two epochs is also consistent with that detected by the EVN. In the final epoch the peak is higher, although we caution that the weak signal-to-noise ratio and the low elevation indicate that this might not be a highly significant discrepancy.

\section{Discussion}

The presence of an AGN within WISE J0716-19 was already implicit in the results presented by K16. They reported an upper limit to the $\mathrm{H} \alpha$ luminosity associated with a star formation rate of $\leq 0.2 M_{\odot} \mathrm{yr}^{-1}$. Based on results reported by Condon (1992), this value corresponds to a radio luminosity $L_{5.0 \mathrm{GHz}} \leq$ $10^{21} \mathrm{~W} \mathrm{~Hz}^{-1}$, about two orders of magnitude lower than that observed at the ATCA quiescence level. Our observations now provide a firm proof of a compact radio source at the centre of WISE J0716-19, with a bolometric radio luminosity of $v L_{v}=5.6 \times 10^{39} \mathrm{erg} \mathrm{s}^{-1}$ that is not variable within the uncertainties. At first sight, this result supports the association proposed by K16 between FRB 150418 and the subsequent episode of variable radio emission.

However, the VLA data (WB16a, angular resolution of $\sim 8^{\prime \prime} \times 3^{\prime \prime}$ at $5.0 \mathrm{GHz}$ ) indicate that variability is still present in the source more than one year after FRB 150418: in Fig. 2 we show the light curve at 5.0 and $5.5 \mathrm{GHz}$ over the time range 2016 February 27 to June 2, obtained with the VLA, EVN, VLBA, and e-MERLIN. The VLA measurements are generally higher and more variable than those obtained with the EVN: the mean flux density and the variability index are $\left\langle S_{\mathrm{VLA}}\right\rangle=195 \mu \mathrm{Jy}$, $V_{\mathrm{VLA}}=0.49$ for the VLA, and $\left\langle S_{\mathrm{VLBI}}\right\rangle=127 \mu \mathrm{Jy}, V_{\mathrm{VLBI}}=0.08$ for the VLBI data (i.e. both EVN and VLBA). We exclude that this discrepancy is due to a secondary variable component in addition to the core imaged with EVN and e-MERLIN. Causality forbids variability on timescales of about a day from diffuse emission resolved out by the EVN and e-MERLIN baselines (scale of $>0.2^{\prime \prime} \sim 1.3 \mathrm{kpc}$ ), which would also be inconsistent with the star formation rates determined by K16. The EVN data themselves do not show evidence for any secondary compact component either, in particular around MJD 57463, when the EVN and VLA data are nearly simultaneous and differ by $150 \mu \mathrm{Jy} \mathrm{beam}^{-1}$; no non-nuclear sources are known to reach such a high luminosity.

This requires us to explore the time, rather than the spatial, domain. It is possible, although unlikely, that the discrepancy is a chance coincidence: a K-S test on the distribution of the VLA and VLBI flux densities provides a probability that the two are drawn from the same distribution as low as 0.011. There is one significant factor to take into account: as a result of the different sensitivity of the two instruments, VLA data are obtained on much shorter timescales (typically $30 \mathrm{~min}$ ) than the EVN data (many hours). We can therefore hypothesise that the parsec-scale source varies on short $(<\mathrm{h})$ timescales, so that the VLA-based light curve resolves the variations, while they are averaged out by the longer EVN observations.

This scenario also presents some challenges. Intrinsic subhour timescale variability from AGNs requires an extremely high brightness temperature, exceeding the inverse-Compton catastrophe limit. On the other hand, WISE J0716-19 is located at low Galactic latitude $(b=-3.2)$, indicating that radio waves are subject to significant refractive scintillation in the ionized interstellar medium of the Milky Way. Akiyama \& Johnson (2016) have argued that the timescale variability of a few days of WISE J0716-19 could indeed be extrinsic if the source had a $T_{\mathrm{b}} \gtrsim 10^{9} \mathrm{~K}$, which is consistent with our result. Scintillation has so far been studied mostly in blazars and little is known about the variability properties of weak sources; however, very rapid variations in WISE J0716-19 would at least be in agreement with the trend of increased variability found for lower flux density sources (Lovell et al. 2008).

Acknowledgements. We thank J. Y. Koay for useful discussions. The European VLBI Network is a joint facility of independent European, African, Asian, and North American radio astronomy institutes. Scientific results from data presented in this publication are derived from the following EVN project codes: RG008A-D. e-MERLIN is a National Facility operated by the University of Manchester at Jodrell Bank Observatory on behalf of STFC. Work by C.C.C. at NRL is supported in part by NASA DPR S-15633-Y.

\section{References}

Akiyama, K., \& Johnson, M. D. 2016, ApJ, 824, L3

Bassa, C., Beswick, R., Tingay, S. J., et al. 2016a, ATel, 8938

Bassa, C. G., Beswick, R., Tingay, S. J., et al. 2016b, MNRAS, 463, L36 Bonetti, L., Ellis, J., Mavromatos, N. E., et al. 2016, Phys. Lett. B, 757, 548 Champion, D. J., Petroff, E., Kramer, M., et al. 2016, MNRAS, 460, L30 Condon, J. J. 1992, ARA\&A, 30, 575

Keane, E. F., Johnston, S., Bhandari, S., et al. 2016, Nature, 530, 453 Liu, T., Romero, G. E., Liu, M.-L., \& Li, A. 2016, ApJ, 826, 82 Lorimer, D. R., Bailes, M., McLaughlin, M. A., et al. 2007, Science, 318, 777 Lovell, J. E. J., Rickett, B. J., Macquart, J.-P., et al. 2008, ApJ, 689, 108 Marcote, B., Giroletti, M., Garrett, M., et al. 2016, ATel, 8865 Spitler, L. G., Scholz, P., Hessels, J. W. T., et al. 2016, Nature, 531, 202 Thornton, D., Stappers, B., Bailes, M., et al. 2013, Science, 341, 53 Tingay, S. J., \& Kaplan, D. L. 2016, ApJ, 820, L31 Vedantham, H. K., Ravi, V., Mooley, K., et al. 2016, ApJ, 824, L9 Williams, P. K. G., \& Berger, E. 2016a, ApJ, 821, L22

Williams, P. K. G., \& Berger, E. 2016b, ATel, 8946

Zhang, B. 2016, ApJ, 822, L14 